\title{
Drug-Induced Liver Injury Caused by Amoxicillin/Clavulanate
}

\author{
Inês Ferreira ${ }^{1}$, Cláudio Gouveia ${ }^{1}$, Carolina Vasques Sr. ${ }^{1}$, Catarina Faria ${ }^{1}$, Ana Pedroso ${ }^{1}$ \\ 1. Internal Medicine, Hospital São Francisco Xavier, Lisboa, PRT
}

Corresponding author: Inês Ferreira, ictgferreira@gmail.com

\begin{abstract}
Drug-induced liver injury (DILI) has a relatively low incidence, and as it is a diagnosis of exclusion, it can become quite a challenge for the clinician. Amoxicillin/clavulanate continues to be one of the most prescribed antibiotics and only rarely causes liver injury. We report a case of DILI associated with this antibiotic to bring attention to a rare side effect of a very commonly prescribed drug. This is the case of a 71-year-old man, with no relevant past medical history, who presented to the Emergency Department due to jaundice in the previous two weeks, with no immediate identifiable cause. The patient was admitted to our Internal Medicine Ward, and after getting a detailed clinical history and excluding other common and severe causes of liver injury, the diagnosis was made that liver injury was due to amoxicillin/clavulanate intake, thus demonstrating the importance of an in-depth history.
\end{abstract}

Categories: Internal Medicine

Keywords: drug, liver, injury, amoxicillin/clavulanate, jaundice

\section{Introduction}

Drug-induced liver injury (DILI) is a rare entity (estimated annual incidence between 10 and 15 per 100,000 persons exposed to prescription medications) [1], which can be classified as hepatocellular, cholestatic, or mixed. Cholestatic DILI is defined as an elevation of alkaline phosphatase greater than twice the normal level and/or an alanine aminotransferase/alkaline phosphatase ratio less than 2. Mixed DILI presents with an alanine aminotransferase/alkaline phosphatase ratio that is greater than 2 but less than 5 , being an intermediate between cholestatic and hepatocellular liver injury [2]. Cholestatic DILI is the most common type in the elderly and takes the longest to resolve after drug suspension. Most cases are mild; however, approximately $10 \%$ of the cases can progress to cirrhosis [2,3]. DILI is a diagnosis of exclusion, resulting in a challenge for the clinician [4].

Review began $12 / 03 / 2020$ Review ended 12/20/2020 Published 12/23/2020

\section{○ Copyright 2020}

Ferreira et al. This is an open access article distributed under the terms of the Creative Commons Attribution License CC-BY 4.0., which permits unrestricted use, distribution, and reproduction in any medium, provided the original author and source are credited.
The traditional diagnostic approach of DILI involves a clinical, laboratorial, and sometimes histological evaluation. It is necessary to establish a time link between drug exposure and the establishment of liver injury, exclude other causes of liver injury, confirm resolution of liver injury after drug suspension, and, in some cases, evaluate the effect of re-exposure. DILI diagnosis can be complex due to the inherent subjectivity of this approach; to solve this problem, diagnostic strategy tools such as the Roussel-Uclaf Causality Assessment Method (RUCAM) were developed [4].

Amoxicillin is a semi-synthetic antibiotic that was combined with clavulanate to prevent degradation by enzymes such as beta-lactamase. Commercialized since 1981, it continues to be one of the most prescribed antibiotics to treat a wide variety of infections. According to a report published by the Centers for Disease Control and Prevention, there were 26.6 million prescriptions of amoxicillin/clavulanate made in 2018, making it the third most prescribed antibiotic in the United States [5].

This case reports an example of jaundice secondary to the use of amoxicillin/clavulanate that was prescribed to treat a respiratory infection. Our aim is to highlight a rare side effect of a very commonly used drug and the associated risk factors. We also wish to draw attention to the fact that the clinical presentation can be quite acute and can occur even after the patient has suspended the antibiotic, which can mislead the clinician into considering obstruction or neoplasms as the most probable diagnosis during the differential diagnosis. Only a complete and in-depth history can avoid unnecessary and invasive examinations.

\section{Case Presentation}

We report the case of a 71-year-old male, with no medical history and no chronic medication. He presented to the Emergency Department complaining of jaundice for two weeks associated with acholia, choluria, pruritus, and a mild rash involving the limbs and torso. He denied having fever, headache, myalgias, arthralgias, respiratory symptoms, abdominal pain, nausea, vomiting, diarrhea, genitourinary symptoms, anorexia, or weight loss. The patient had no known allergies, and there was no exposure to alcohol, tobacco, or any illicit drugs. No relevant family history findings were present. 


\section{Cureus}

On physical examination, the patient presented with no fever and hemodynamic stability. The skin and sclera showed visible jaundice, and a generalized, mild, macular, non-petechial rash was also present. Abdominal examination showed a completely normal, painless abdomen, without hepatosplenomegaly or palpable masses, without any signs of chronic liver disease or portal hypertension. Neurological examination was completely normal. Multiple medical tests were performed to determine the cause of jaundice. Laboratory analysis (Table 1) revealed a cholestatic pattern with hyperbilirubinemia caused by elevated levels of conjugated bilirubin. Abdominal ultrasound and computerized tomography (CT) scan showed a liver with normal dimensions, with no structural anomalies or lesions, a gallbladder with no evidence of gallstones, and bile ducts with maintained caliber. There were no relevant findings concerning the pancreas.

\begin{tabular}{|c|c|c|}
\hline & Relevant Laboratorial Results & Reference Values \\
\hline Hemoglobin & $13.6 \mathrm{~g} / \mathrm{dL}$ & $13.0-17.0 \mathrm{~g} / \mathrm{dL}$ \\
\hline Mean Corpuscular Volume & $86.1 \mathrm{fL}$ & 80.0-96.1 fL \\
\hline Mean Corpuscular Hemoglobin & $28.0 \mathrm{pg}$ & 27.3-33.7 pg \\
\hline Leucocytes & $6.2 \times 10^{9} / \mathrm{L}$ ( $0 \%$ eosinophils) & $4.0-10.0 \times 10^{9} / \mathrm{L}$ \\
\hline Platelets & $306 \times 10^{9} / \mathrm{L}$ & $150-400 \times 10^{9} / \mathrm{L}$ \\
\hline Prothrombin Time & 11.6 seconds & $<14$ seconds \\
\hline Urea & 27 mg/dL & $17-49 \mathrm{mg} / \mathrm{dL}$ \\
\hline Creatinine & 0.76 mg/dL & $0.7-1.20 \mathrm{mg} / \mathrm{dL}$ \\
\hline Sodium & $140 \mathrm{mmol} / \mathrm{L}$ & $136-145$ mmol/L \\
\hline Potassium & $4.66 \mathrm{mmol} / \mathrm{L}$ & 3.5-5.10 mmol/L \\
\hline Albumin & $4.1 \mathrm{~g} / \mathrm{dL}$ & $3.5-5.2 \mathrm{~g} / \mathrm{dL}$ \\
\hline Total Bilirubin & $8.79 \mathrm{mg} / \mathrm{dL}$ & $<1.40 \mathrm{mg} / \mathrm{dL}$ \\
\hline Conjugated Bilirubin & $6.21 \mathrm{mg} / \mathrm{dL}$ & $<0.3 \mathrm{mg} / \mathrm{dL}$ \\
\hline Alanine Transaminase & $193 \mathrm{U} / \mathrm{L}$ & $<41 \mathrm{U} / \mathrm{L}$ \\
\hline Aspartate Transaminase & 69 U/L & $<40 \mathrm{U} / \mathrm{L}$ \\
\hline Gamma-Glutamyltransferase & 190 U/L & 10-71 U/L \\
\hline Alkaline Phosphatase & $262 \mathrm{U} / \mathrm{L}$ & 40-130 U/L \\
\hline Lactate Dehydrogenase & $262 \mathrm{U} / \mathrm{L}$ & $135-225 \mathrm{U} / \mathrm{L}$ \\
\hline Lipase & 19 U/L & 13-60 U/L \\
\hline Thyroid-Stimulating Hormone & $1.0 \mathrm{uUl} / \mathrm{mL}$ & $0.27-4.20 \mathrm{uUI} / \mathrm{mL}$ \\
\hline C-Reactive Protein & 1.75 mg/dL & $<0.5 \mathrm{mg} / \mathrm{dL}$ \\
\hline
\end{tabular}

Urinalysis: Absence of proteins, glucose, ketone bodies, urobilinogen, and hemoglobin. Bilirubin (+).

Serology panel: Hepatitis A virus immunoglobulin M, hepatitis B surface antigen, and hepatitis C virus antibodies were negative. AntiHIV antibodies were also negative.

TABLE 1: Blood and urine laboratorial results in the Emergency Department.

Due to the need for further investigation, the patient was admitted to the Internal Medicine Ward. A more detailed clinical history acquired during the hospital stay revealed that the patient had been taking amoxicillin/clavulanate $875 / 125 \mathrm{mg}$ twice a day for a respiratory infection for 14 days. Two days after completing the antibiotic scheme, the patient presented with mild jaundice of the scleral conjunctiva (16 days after treatment with amoxicillin/clavulanate was initiated), which progressed over the next two weeks, motivating the patient to seek medical help in the Emergency Department. 


\section{Cureus}

Even though there was a temporal link between the drug and the development of liver injury, DILI is a diagnosis of exclusion, and due to the patient's age and the exuberance of the analytical findings, other causes of cholestasis had to be excluded. The differential diagnosis of hyperbilirubinemia due to elevated conjugated bilirubin must take into account intra- and extrahepatic cholestasis. For this reason, during the hospital stay, the patient was submitted to various tests, including immunological investigation (Table 2), iron studies (Table 3), and additional serology (Table 4).

\begin{tabular}{|c|c|c|}
\hline & Results & Reference Values \\
\hline Rheumatoid Factor (U/mL) & 10 & $<15$ \\
\hline Antinuclear Antibodies & Negative & \\
\hline Extractable Nuclear Antigen Antibodies & Negative & \\
\hline Anti-Mitochondrial Antibodies & Negative & \\
\hline Anti-Smooth Muscle Antibodies & Negative & \\
\hline Anti-Soluble Liver Antigen Antibodies & Negative & \\
\hline Anti-Neutrophil Cytoplasmic Antibodies & Negative & \\
\hline $\operatorname{lgG}(\mathrm{mg} / \mathrm{dL})$ & 1640 & $600-1500$ \\
\hline IgG Subclass 1 (mg/dL) & 977 & $422-1290$ \\
\hline IgG Subclass 2 (mg/dL) & 883 & $117-747$ \\
\hline IgG Subclass 3 (mg/dL) & 151 & $40-130$ \\
\hline IgG Subclass 4 (mg/dL) & 25 & $1-291$ \\
\hline Immunoglobulin M (mg/dL) & 144 & $50-300$ \\
\hline Immunoglobulin A (mg/dL) & 610 & $50-400$ \\
\hline
\end{tabular}

TABLE 2: Immunology panel with no relevant findings, thus eliminating an autoimmune cause for the liver injury.

IgG, immunoglobulin G

\begin{tabular}{|c|c|c|}
\hline Iron Studies & Results & Reference Values \\
\hline Iron (ug/dL) & 70 & 33-193 \\
\hline Ferritin (ng/mL) & 206 & $18-464$ \\
\hline Total Iron-Binding Capacity (ug/dL) & 261 & $250-425$ \\
\hline Transferrin Saturation (\%) & 27 & $20-55$ \\
\hline
\end{tabular}

\section{TABLE 3: Iron studies with no relevant alterations.}




\section{Cureus}

\begin{tabular}{|c|c|c|}
\hline Virus & IgG & $\lg M$ \\
\hline Cytomegalovirus & Negative & Negative \\
\hline Epstein-Barr & Positive & Negative \\
\hline Varicella Zoster & Negative & Negative \\
\hline Herpes Simplex Type 1/2 & Negative & Negative \\
\hline Hepatitis E & Negative & Negative \\
\hline
\end{tabular}

TABLE 4: Continuation of serological investigation that was started in the Emergency Department and was completed during the hospital stay.

IgG, immunoglobin G; IgM, immunoglobulin M

There was no sign of infection, neoplasm, and autoimmune or metabolic disease either clinically, biochemically, or radiologically (serological panel was negative for hepatitis A, B, C, E, cytomegalovirus, Epstein-Barr virus, herpes simplex virus, and varicella zoster virus; serological levels of IgG4 were normal; antibodies for primary biliary cholangitis were negative; thyroid function was normal; iron studies were normal). Through abdominal imaging with magnetic resonance cholangiopancreatography, we were also able to confirm that the liver, gallbladder, and pancreas had conserved dimensions, with no identifiable nodular lesions, and eliminate the possibility of intra- or extrahepatic obstruction. In light of the patient's age, absence of family history, and lack of clinical, biochemical, and radiological support, the diagnosis of hereditary and infiltrative diseases was not pursued, and hence the decision to not perform liver biopsy.

The patient was discharged at the seventh day of admission. No specific treatment was given apart from fluid therapy and occasionally antihistaminic drugs for pruritus.

Through the patient's follow-up we were able to confirm the resolution of all the findings previously mentioned after a five-month period (<180 days) since antibiotic suspension (Figures 1,2). Using the RUCAM scale our case scored nine points, making the DILI diagnosis very probable [6].

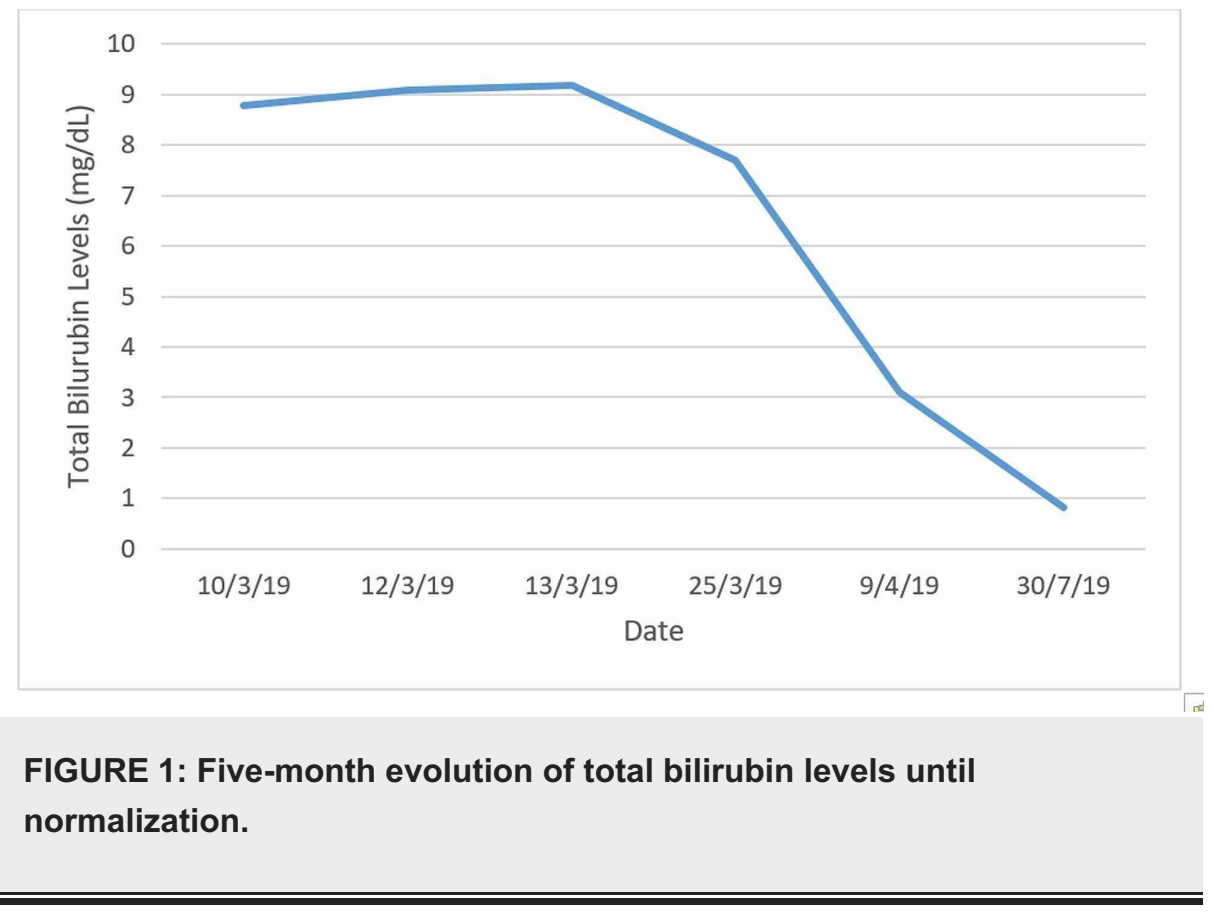




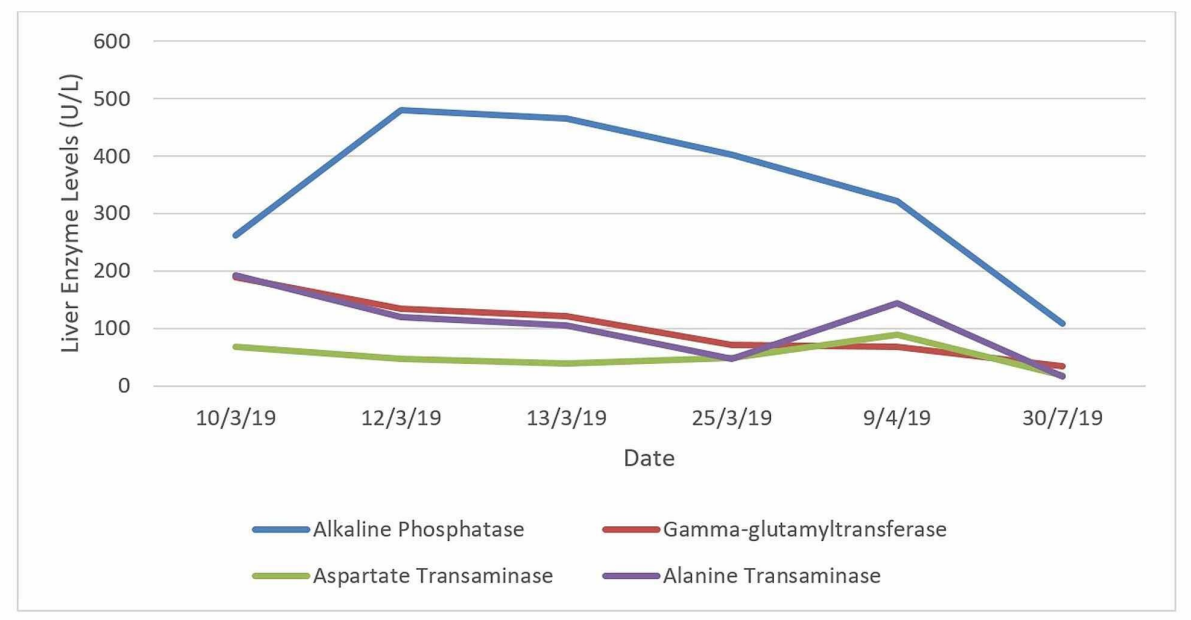

FIGURE 2: Five-month evolution of liver enzymes until normalization.

Discussion

We believe that it is important to report this case due to the fact that amoxicillin/clavulanate is a very frequent and widely used antibiotic [7]. The incidence of amoxicillin/clavulanate-induced liver injury is greater than that induced by amoxicillin alone, and although it can cause any type of DILI, cholestasis is the most frequent kind of presentation $[7,8]$.

The pathogenesis behind amoxicillin/clavulanate liver injury requires more studies; however, it is believed that immuno-allergic mechanisms are involved. The presence of eosinophils in the inflammatory infiltrate, hypersensitivity manifestations such as skin rashes and hyper-eosinophilia, and the association with human leucocyte antigen (HLA) class II genes (DRB1 * 1501-DRB5 * 0101-DQB1 * 0602) reinforce the hypothesis that an immune aggression is involved in the liver injury caused by this antibiotic [7-9]. Risk factors include male sex, alcohol consumption, repeated use of the drug, concomitant use of other hepatotoxic drugs, and age over 55 years $[7,8,10]$. The duration of treatment was also included as a risk factor in some reviews [7].

Clinical manifestations are predominantly cholestatic, namely anorexia, nausea, vomiting, jaundice, choluria, acholia, pruritus, and, occasionally, painful hepatomegaly. Hypersensitivity manifestations, such as skin rash and fever, can sometimes occur [7-9]. DILI can be considered up to 90 days after initial drug intake or up to 30 days after drug suspension. This clinical condition can take up to six months after drug suspension to completely resolve [6]. Even though most cases have a benign course, on rare occasions it can progress to liver failure, requiring close monitoring $[7,8]$. Treatment consists of withdrawal of the offending drug and supportive treatment such as fluid replacement. Cholestatic symptoms can become very limiting and demand treatment with antiemetic and analgesic drugs, as well as cholestyramine, antihistamine, ursodeoxycholic acid, or even sertraline to control pruritus, depending on its severity. Due to the fact that an immune mechanism might be involved in DILI, corticotherapy has been suggested, but there are no supporting data that suggest a reduction in morbidity [11].

This case describes a 71-year-old man who started to manifest signs of cholestatic jaundice two days after suspending treatment with amoxicillin/clavulanate after a 14-day treatment. The patient developed acute conjugated hyperbilirubinemia with a cholestatic pattern of the liver function tests, however with no other severity criteria of impending severity. The complete clinical and biochemical resolution lasted five months, with no specific treatment. Several risk factors for DILI were present in this case, namely, advanced age, male sex, and prolonged exposure to the drug. A complete anamnesis and extensive biochemical and radiological examinations excluded other common causes of liver injury. RUCAM score was applied (nine points), making DILI highly probable. A relevant factor, which complicated the diagnosis, was that the patient denied recent intake of drugs when initially interviewed. Liver biopsy was not performed since other common causes of liver injury were excluded, making DILI secondary to amoxicillin/clavulanate the most probable diagnosis. When DILI is the most probable diagnosis and other causes of liver injury have been eliminated, the best course of action is vigilance and monitoring, certifying a decrease in liver enzyme levels, and thus sparing the patient from painful and invasive procedures. Exposure to amoxicillin/clavulanate should be avoided in the future $[7,8]$.

\section{Conclusions}

This clinical report demonstrates the importance of a complete and detailed patient history and a responsible use of antibiotics. This is especially important in at-risk groups, a population that is a frequent 
user of health services and is more susceptible to infection.

As amoxicillin/clavulanate is a very commonly used antibiotic, reports such as this one are fundamental to highlight this rare side effect so that medical professionals become more aware of its existence. By doing so, we hope to spare patients of unnecessary examinations, which can be invasive and with potential serious complications.

\section{Additional Information \\ Disclosures}

Human subjects: Consent was obtained by all participants in this study. Conflicts of interest: In compliance with the ICMJE uniform disclosure form, all authors declare the following: Payment/services info: All authors have declared that no financial support was received from any organization for the submitted work. Financial relationships: All authors have declared that they have no financial relationships at present or within the previous three years with any organizations that might have an interest in the submitted work. Other relationships: All authors have declared that there are no other relationships or activities that could appear to have influenced the submitted work.

\section{References}

1. Bell LN, Chalasani N: Epidemiology of idiosyncratic drug-induced liver injury. Semin Liver Dis. 2009, 29:337-47. 10.1055/s-0029-1240002

2. Bjornsson ES, Jonasson JG: Drug-induced cholestasis. Clin Liver Dis. 2013, 17:191-209. 10.1016/j.cld.2012.11.002

3. Andrade RJ, Lucena MI, Kaplowitz N, et al.: Outcome of acute idiosyncratic drug-induced liver injury: longterm follow-up in a hepatotoxicity registry. Hepatology. 2006, 44:1581-8. 10.1002/hep.21424

4. Rockey DC, Seeff LB, Rochon J, et al.: Causality assessment in drug-induced liver injury using a structured expert opinion process: comparison to the Roussel-Uclaf Causality Assessment Method. Hepatology. 2010, 51:2117-26. 10.1002/hep.23577

5. Centers for Disease Control and Prevention. Outpatient antibiotic prescriptions - United States, 2018 Accessed: December 22, 2020: http://www.cdc.gov/antibiotic-use/community/pdfs/Annual-Report-2018H.pdf.

6. Danan G, Teschke R: RUCAM in drug and herb induced liver injury: the update . Int J Mol Sci. 2016, 17:14. 10.3390/ijms 17010014

7. Berg P, Hahn EG: Hepatotoxic reactions induced by beta-lactamase inhibitors . Eur J Med Res. 2001, 6:53542.

8. deLemos AS, Ghabril M, Rockey DC, et al.: Amoxicillin-clavulanate-induced liver Injury. Dig Dis Sci. 2016, 61:2406-16. 10.1007/s10620-016-4121-6

9. O’Donohue J, Oien KA, Donaldson P, Underhill J, Clare M, MacSween RN, Mills PR: Co-amoxiclav jaundice: clinical and histological features and HLA class II association. Gut. 2000, 47:717-20. 10.1136/gut.47.5.717

10. Lucena MI, Andrade RJ, Kaplowitz N, et al.: Phenotypic characterization of idiosyncratic drug-induced liver injury: the influence of age and gender. Hepatology. 2009, 49:2001-9. 10.1002/hep.22895

11. Herrero-Herrero JI, García-Aparicio J: Corticosteroid therapy in a case of severe cholestasic hepatitis associated with amoxicillin-clavulanate. J Med Toxicol. 2010, 6:420-3. 10.1007/s13181-010-0019-4 